\title{
Fading Effects on the Lower Shifting of Mode Switching Thresholds in the Rate Adaptive IEEE 802.11a/g WLANs
}

\author{
Chie Dou, Li-Shin Wang \\ Department of Electrical Engineering, National Yunlin University of Science and Technology, Touliu, Taiwan, China \\ E-mail: \{douc,g9851710\}@yuntech.edu.tw \\ Received May 3, 2010; revised June 29, 2010; accepted August 2, 2010
}

\begin{abstract}
In this paper we used the probability distribution of the average channel gain of the fading channel to analyze the degree of fading effects on both the PER (packet error rate) and the throughput in OFDM systems. Instead of solely examining the average received SNR (signal-to-noise ratio) value of a packet, considering the whole distribution of the average received SNR allows us to aggregate a better selection of the mode switching thresholds in the rate adaptive $802.11 \mathrm{a} / \mathrm{g}$ WLAN. This paper demonstrates that the set of mode switching thresholds can be determined for each individual target $E_{b} / N_{0}$, so that the optimal throughput performance is obtained on a per target $E_{b} / N_{0}$ basis. Numerical results show that mode switching thresholds should be reduced with the lowering of target $E_{b} / N_{0}$ values. This conclusion could have significant implications for improving the performances of location (distance)-dependent mobile applications, since the determinations of target $E_{b} / N_{0}$ values are closely related to the distances between mobile devices and the access point.
\end{abstract}

Keywords: 802.11a/g, Fading Channels, Packet Error Rate, Channel Gain, Link Adaptation

\section{Introduction}

THE IEEE $802.11 \mathrm{a} / \mathrm{g}$ WLAN is an OFDM (orthogonal frequency division multiplexing)-based basic service set (BSS) in which stationary devices and an access point (AP) communicate. The multipath channel effect causes a communicating pair of devices to experience a particular fading realization which may be different from those of other communicating pairs in the BSS. The transmiting device determines a target signal quality by setting an initial SNR or a target $E_{b} / N_{0}$, so that a certain qualityof-service (QoS) may be satisfied at the receiver side. The determination of the target signal quality is often dependent on the distance between the pair of communicating devices. An example of the correspondence between the distance and the initial SNR under 802.11 a standard [1] was given in [2]. In this paper, we first determine the target $E_{b} / N_{0}$, which can subsequently be used to directly calculate the initial SNR value per subcarrier for different rate modes. Furthermore, we clarify the relationship between the target $E_{b} / N_{0}$ and the received SNR of the packet in the rate adaptive IEEE $802.11 \mathrm{a} / \mathrm{g} \mathrm{WL}$ AN over frequency selective fading channels. In OFDM systems, a carrier frequency offset (CFO) can give rise to amplitude reduction and phase rotation of the desired signal, inducing inter-carrier interference (ICI) [3]. Consequently, the signal-to-interference-and-noise ratio (SINR) should be considered at the receiver $[4,5]$. The SINR for frequency selective fading channels with a CFO has already been investigated [4]. Evidently the average SINR will be reduced to the average SNR if the ICI can be ignored. In this paper, we assumed conditions of perfect synchronization with no timing and frequency offsets. Thus, we examine the received SNR of each subcarrier and the average received SNR of a packet instead of the SINR.

The channel transfer functions (or channel gains) of fading channels significantly impact both the received SNR and the PER of received packets in OFDM-based WLANs $[2,6-8]$. An analytical PER calculation method was developed in [2] for OFDM-based systems using a hard decoder. This paper also derives the PER expression for convolutional-coded hard-decision decoded OFDM systems on a fading realization basis. The PER calculation method presented in this paper differs from that of [2] by introducing a more simple yet effective analysis process in that both methods provide only an analytical upper bound. The average PER expression captures the PER versus initial SNR for all data rates in [2]. However, 
it does not provide insight into the influence that the distribution of the channel transfer function (or channel gain) over the subcarriers has on the PER. This paper uses the probability distribution of the average channel gain of the fading channel to analyze the degree of fading effects on both the PER and the throughput in OFDM systems. The average channel gain of a fading realization is obtained by averaging the channel gains over all subcarriers. A similar definition of the average channel gain can be found in [9]. Estimation schemes based on an "indicator" concept obtain accurate predictions of the PER via evaluations of the channel transfer function [6,7]. The estimated variance of the transfer function amplitude serves as a simple but effective indicator. However, indicatoraided SNR estimations and direct PER predictions must take into account some complex optimizations of parameters that may cause errors in channel estimation. A conventional link adaptation algorithm takes the measured SNR as the only input from the PHY layer; there exists the possibility that it ignores the stochastic variability of the multipath fading channel and does not exploit the full potential of the link adaptation [6,7]. Instead of solely examining the average received SNR value of a packet, this paper considers the whole distribution of the average received SNR in a way that facilitates a better performance of link adaptation by exploiting the stochastic variability of the multipath fading channel. Previously, two analytical methods have been presented for estimating the bit error rate (BER) of coded multicarrier systems operating over frequency-selective quasi-static channels with non ideal interleaving [8]. However, explicit knowledge of Rayleigh-distributed frequency-domain subcarrier channel gains and their correlation matrices are prerequisites for finding the BER.

In this paper, the average PER of individual average received SNR value is obtained for different rate modes both by an analytical approach and by the simulation on a per target $E_{b} / N_{0}$ basis. The influences of the probability distribution of average channel gain on the average PER are investigated and compared between ETSI (European telecommunications standards institute) BRAN A and $\mathrm{C}$ channel models [10]. Performance result of the average PER shows that under a given rate mode, better PER performance is obtained with the same average received SNR by lowering the target $E_{b} / N_{0}$ value. This observation has significant impact on the determination of mode switching thresholds in $802.11 \mathrm{a} / \mathrm{g}$ link adaptation.

For conventional link adaptation techniques, a set of mode switching thresholds for the received SNR is chosen for different rate modes based on the achieved throughput taking the PER for each rate mode into account $[11,12]$ or based on the delay performance of a certain target PER for all rate modes [13]. Unlike conventional link adaptation techniques, in this paper an optimal set of mode switching thresholds is determined for each target $E_{b} / N_{0}$. Numerical results show that mode switching thresholds should be shifted downwards with the lowering of target $E_{b} / N_{0}$ values. This conclusion is useful in improving the performances of location (distance)-dependent mobile applications, such as distributed camera network (DCN). In a DCN, digital cameras using 802.11 protocol for video transmissions may have different target $E_{b} / N_{0}$ values according to the distances between their locations and the access point. The performances of video transmissions in a DCN can be improved if each particular digital camera can use the optimal set of mode switching thresholds determined by the corresponding target $E_{b} / N_{0}$.

The rest of this paper is organized as follows. Section 2 presents the channel fading effects in OFDM-based systems. The impact of channel gains over subcarriers on the average received SNR of a received packet is investigated. For the rate adaptive $802.11 \mathrm{a} / \mathrm{g}$ WLAN, an analytical approach is proposed to obtain the average PER of individual average received SNR value for different rate modes on a per target $E_{b} / N_{0}$ basis. Instead of just looking at the average received SNR value of a packet, we consider the whole distribution of the average received SNR, which leads to a better average PER and also throughput performances. Section 3 presents the simulation model and the channel estimation technique. The average PER derived from the analytical upper bound is compared to the simulation. Numerical results and their applications to the throughput-based link adaptation in $802.11 \mathrm{a} / \mathrm{g}$ WLAN are presented in Section 4. We demonstrate that the set of mode switching thresholds can be determined for each individual target $E_{b} / N_{0}$ for optimal throughput performance. Numerical results show that mode switching thresholds should be reduced with the lowering of target $E_{b} / N_{0}$ values. Finally, Section 5 states the conclusions.

\section{Fading Effects in OFDM-Based Systems}

Figure 1 shows a block diagram of OFDM-based systems using $N$-point IFFT/FFT in an equivalent low-pass

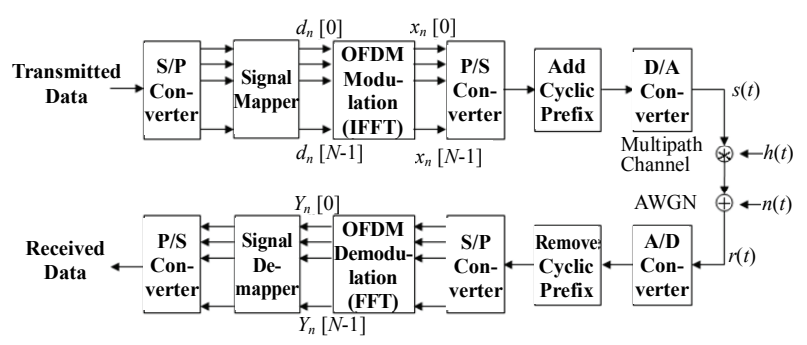

Figure 1. Block diagram of OFDM-based systems in an equivalent low-pass system. 
system. Basically, in an OFDM system the serial bit stream is transformed to a parallel form. The bits to be transmitted are first mapped onto constellation points with the $M$-ary PSK (phase shift keying) or QAM (quadrature amplitude modulation) scheme, then those parallel data are modulated by means of an IFFT (inverse fast Fourier transform) on $N$ parallel subcarriers. In 802.11a, different combinations of the code rate and modulation type results in eight rate modes specified in the standard [1]. These rate modes and their corresponding code rates and modulation types and coded bits per subcarrier are listed in Table 1. Let $d_{n}[i]$ be the modulation symbol of the $i$-th subcarrier for the nth OFDM symbol. Without timing and frequency offset, the baseband discrete-time data signal of the $k$-th sample of the $n$-th transmitted OFDM symbol can be given by

$$
x_{n}[k]=\sum_{i=0}^{N-1} d_{n}[i] \cdot e^{j 2 \pi k i / N}, \quad k=0,1, \ldots, N-1 .
$$

The output signal $s(t)$ of the transmitter traverses through a multipath channel. To accurately measure the delay and fading caused by multipath, the Naftali model, which is a consistent channel model to compare different WLAN systems in an indoor radio environment is commonly used [14]. Using Naftali model, we can compose the channel impulse response of complex samples using random uniformly distributed phase and Rayleigh distributed magnitude. We assume the time-varying channel consists of $L$ multipath components, and each path component is characterized by an amplitude $h_{l}$ and a delay time $\tau_{l}$. The model has the form

$$
h(t)=\sum_{l=0}^{L-1} h_{l} \delta\left(t-\tau_{l}\right) .
$$
by

The channel impulse response of the $l$-th path is given

$$
h_{l}=N\left(0, \frac{1}{2} \sigma_{l}^{2}\right)+j N\left(0, \frac{1}{2} \sigma_{l}^{2}\right),
$$

where $N\left(0, \sigma_{l}^{2} / 2\right)$ is a Gaussian random variable with zero mean and variance $\sigma_{l}^{2} / 2$, where

$$
\sigma_{l}^{2}=\sigma_{0}^{2} \cdot e^{-\tau_{l} / T_{R M S}},
$$

where $\sigma_{0}^{2}$ is selected so that the summation of all $\sigma_{l}^{2}$ must be normalized to one to ensure the average received power be the same. Here $T_{R M S}$ is the root mean square delay spread of the channel response. Due to the exponential decaying term expressed in (4), the Naftali model is usually called exponential channel model.

After the multipath channel, the received signal $r(t)$ is further corrupted by an Additive White Gaussian Noise (AWGN) as follows:

$$
r(t)=s(t) * h(t)+n(t)
$$

Table 1. Rate mode dependent parameters in $802.11 \mathrm{a} / \mathrm{g}$ standard.

\begin{tabular}{ccccc}
\hline $\begin{array}{c}\text { Rate mode } \\
(R M)\end{array}$ & $\begin{array}{c}\text { Data rate } \\
(\mathrm{Mb} / \mathrm{s})\end{array}$ & $\begin{array}{c}\text { Modulation } \\
\text { type }\end{array}$ & $\begin{array}{c}\text { Coding rate } \\
(C R)\end{array}$ & $\begin{array}{c}\text { Coded bits per } \\
\text { subcarrier }\left(N_{\mathrm{BPSC}}\right)\end{array}$ \\
\hline 1 & 6 & BPSK & $1 / 2$ & 1 \\
2 & 9 & BPSK & $3 / 4$ & 1 \\
3 & 12 & QPSK & $1 / 2$ & 2 \\
4 & 18 & QPSK & $3 / 4$ & 2 \\
5 & 24 & 16-QAM & $1 / 2$ & 4 \\
6 & 36 & 16-QAM & $3 / 4$ & 4 \\
7 & 48 & 64-QAM & $2 / 3$ & 6 \\
8 & 54 & 64-QAM & $3 / 4$ & 6 \\
\hline
\end{tabular}

where ' $*$ ' represents the convolution operation and $n(t)$ is AWGN with the two-side power spectral density, $N_{0} / 2$.

At the receiver, the received signal is down-converted to the baseband signal and enters FFT. In this process, we assume that the synchronization in frequency and timing are perfect and the delay spread is smaller than the guard interval (GI), so that ICI and ISI (inter symbol interference) are ignored. After taking an $N$ point FFT on the nth OFDM symbol, we have the received signal for subcarrier $i$ over a slowly time-varying time-dispersive channel given by

$$
Y_{n}[i]=d_{n}[i] H_{i}+Z_{n}[i],
$$

where $H_{i}$ is the channel transfer function of subcarrier $i$ in frequency domain and $Z_{n}[i]$ is the noise term of the $i$-th subcarrier due to AWGN. The channel frequency response $H_{i}$ can be given by [15-17]:

$$
H_{i}=\sum_{l=0}^{L-1} h_{l} \cdot e^{\frac{-j 2 \pi i \tau_{l}}{N T_{s}}}, i=0,1, \ldots, N-1,
$$

where $1 / T_{s}$ is the total bandwidth of the system. In the IEEE $802.11 \mathrm{a} / \mathrm{g}$ standard, the total bandwidth of the system is $20 \mathrm{MHz}$.

\subsection{Impact of Channel Gains on Received SNR}

For different rate modes of $802.11 \mathrm{a} / \mathrm{g}$, the initial SNR value of subcarrier $i$ can be given by

$$
S N R_{S C_{i}}=10^{\frac{\left(E_{b} / N_{0}\right)}{10}} \times C R \times N_{B P S C},
$$

where $E_{b}$ is the transmitted signal energy per bit, and $C R$ is the code rate of the given rate mode and $N_{B P S C}$ is the corresponding coded bits per subcarrier as listed in Table 1. From (8), it is clear that the initial SNR value of subcarrier $i$ will not be the same for different rate modes of $802.11 \mathrm{a} / \mathrm{g}$ under the same $E_{b} / N_{0}$, due to different code rates and number of coded bits per subcarrier. If we further assume that the average energy of an OFDM symbol is equal to 1 and the initial SNR values of all 
subscribers are equal, then the average energy of the signal constellation for each subcarrier is equal to $1 / N(N$ $=64)$, and the initial SNR value per subcarrier, denoted by $\eta$, can be defined as

$$
\eta \equiv S N R_{S C_{i}}=\frac{1 / N}{N_{0}}=\frac{1}{N \times 2 \sigma_{Z}^{2}},
$$

where $\sigma_{Z}^{2}=N_{0} / 2$ is the noise variance of AWGN.

From (8) and (9), the $E_{b} / N_{0}$ can be expressed as

$$
\frac{E_{b}}{N_{0}}=10 \cdot \log _{10} \frac{1}{N \times C R \times N_{B P S C} \times\left(2 \sigma_{Z}^{2}\right)} .
$$

From (10) it shows that when $E_{b} / N_{0}$ is the same, the rate mode should be decreased if the variance of AWGN increases. For different rate modes, noise power can be calculated to generate AWGN that will be used in the simulation model presented in the next section.

Let $\gamma_{i}$ be the received SNR of the $i$-th subcarrier, which is dependent on the initial SNR value of the $i$-th subcarrier in the transmitter side and the channel gain $\left|H_{i}\right|^{2}$ in frequency domain. It can be given by [18,19]:

$$
\gamma_{i}=\left|H_{i}\right|^{2} S N R_{S C_{i}}=\left|H_{i}\right|^{2} \eta \text {. }
$$

In [1], the $802.11 \mathrm{a} / \mathrm{g}$ system uses 52 subcarriers that are modulated using $M$-ary PSK or QAM scheme, including 48 data subcarriers and 4 pilot subcarriers. Let $\bar{\gamma}$ be the average received SNR after demodulation over all these 52 subcarriers and $I$ be the set of coefficient indices corresponding to these 52 subcarriers. We have

$$
\bar{\gamma}=|\bar{H}|^{2} \eta
$$

where $|\bar{H}|^{2}$ is the average channel gain over all subcarriers [9]:

$$
|\bar{H}|^{2}=\frac{1}{52} \sum_{\text {all } i \in I}\left|H_{i}\right|^{2} .
$$

If we assume the channel is static for each transmitted packet (that is, the channel gain is the same for a given subcarrier between consecutive OFDM symbols), then the average received SNR of the packet is also $\bar{\gamma}$ given by (12).

\subsection{Fading Realization of Channel Models}

In this paper, PER performance comparisons are made between ETSI/BRAN A and C channel models. Fading realizations of both channel models are discussed in this subsection. ETSI/BRAN A channel (18-ray) was defined to represent a typical small office NLOS (non line-of-sight) indoor environment with a small RMS delay spread (50 ns). ETSI/BRAN C channel (18-ray) was defined to represent a typical large office NLOS indoor environment with a large RMS delay spread (150 ns). The power delay profile (PDP) of ETSI/BRAN A and C channels are shown in Table 2 and Table 3, respectively.

Since BRAN A and C channel models are not truly exponential channel models, the variance $\sigma_{l}^{2} / 2$ of the Gaussian random variable $N\left(0, \sigma_{l}^{2} / 2\right)$ used in (3) could not be calculated by (4) directly. In this study, we use the power level (in dB) specified for each ray in the PDP of the channel model to calculate the desired $\sigma_{l}^{2}$. First, the decibel measure for each ray is converted to its relative power ratio, for example, $0 \mathrm{~dB}$ is converted to power ratio 1 . Let $v_{l}^{2}$ denote the relative power ratio converted from the power level $(\mathrm{dB})$ specified for the $l$-th ray. Since the summation of all $\sigma_{l}^{2}$ must be normalized to one to ensure the average received power is the same thus we have

$$
\sigma_{l}^{2}=v_{l}^{2} / \sum_{k=1}^{L-1} v_{k}^{2} .
$$

Now, we can use (3) and (14) to generate the channel impulse response of the $l$-th path for BRAN A and C channel models. Fading realizations of both channel models are generated by (7) and the average channel gain of each fading realization is give by (13).

Figure 2 shows the probability distribution of average channel gain over 100,000 samples of fading realization

Table 2. Power delay profile of BRAN A channel.

\begin{tabular}{cccc}
\hline Delay $(\mu \mathrm{s})$ & Power $(\mathrm{dB})$ & Delay $(\mu \mathrm{s})$ & Power $(\mathrm{dB})$ \\
\hline 0.00 & 0.00 & 0.09 & -7.80 \\
0.01 & -0.90 & 0.11 & -4.70 \\
0.02 & -1.70 & 0.14 & -7.30 \\
0.03 & -2.60 & 0.17 & -9.90 \\
0.04 & -3.50 & 0.20 & -12.5 \\
0.05 & -4.30 & 0.24 & -13.7 \\
0.06 & -5.20 & 0.29 & -18.0 \\
0.07 & -6.10 & 0.34 & -22.4 \\
0.08 & -6.90 & 0.39 & -26.7 \\
\hline
\end{tabular}

Table 3. Power delay profile of BRAN C channel.

\begin{tabular}{cccc}
\hline Delay $(\mu \mathrm{s})$ & Power $(\mathrm{dB})$ & Delay $(\mu \mathrm{s})$ & Power $(\mathrm{dB})$ \\
\hline 0.00 & -3.30 & 0.23 & -3.00 \\
0.01 & -3.60 & 0.28 & -4.40 \\
0.02 & -3.90 & 0.33 & -5.90 \\
0.03 & -4.20 & 0.40 & -5.30 \\
0.05 & 0.00 & 0.49 & -7.90 \\
0.08 & -0.90 & 0.60 & -9.40 \\
0.11 & -1.70 & 0.73 & -13.2 \\
0.14 & -2.60 & 0.88 & -16.3 \\
0.18 & -1.50 & 1.05 & -21.2 \\
\hline
\end{tabular}


for two channel models. A rectangle-based integration method with equally spaced "bins" has been employed to derive the curves. Here the width of each "bin" is 0.05 . From Figure 2, it shows that the mean value of both probability distributions is equal to one. We see that for small $|\bar{H}|^{2}$, say smaller than 0.5, BRAN A channel has higher probability distribution than that of BRAN $\mathrm{C}$ channel. For instance, it gives $\operatorname{Pr}\left(|\bar{H}|^{2} \leq 0.5\right)=0.2316$ for BRAN A channel and $\operatorname{Pr}\left(|\bar{H}|^{2} \leq 0.5\right)=0.0913$ for BRAN C channel. For large $|\bar{H}|^{2}$, say larger than 2, BRAN A channel also has higher probability distribution than that of BRAN C channel. For instance, it gives $\operatorname{Pr}\left(|\bar{H}|^{2} \geq 2\right)=0.0788$ for BRAN A channel and $\operatorname{Pr}\left(|\bar{H}|^{2} \geq 2\right)=0.0258$ for BRAN C channel. The impacts of $|\bar{H}|^{2}$ in its full statistics on the PER performance under BRAN A and $\mathrm{C}$ channel models are discussed in the following.

Figure 3 shows the probability distribution of average received SNR under two channel models given that the rate mode $R M=3$ and the target $E_{b} / N_{0}$, denoted by $T$, is $16 \mathrm{~dB}$. Rectangle-based integration method with equally spaced "bins" has also been employed to derive the probability distribution of average received SNR. For a particular integer-valued average received SNR, $R$, the range of the corresponding sampling bin is $(R-0.5, R+$ $0.5]$. In this paper, only integer-valued average received SNR is considered. However, fractionary average received SNR can be treated by reducing the width of each sampling bin. From (8) and (12), the average received SNR of a packet for a certain rate mode can also be written as

$$
\bar{\gamma}=|\bar{H}|^{2} T+10 \log _{10}\left(C R \times N_{B P S C}\right)
$$

Because of the code rate multiplied by the coded bits per subcarrier is 1 for $R M=3$, a simple relationship $\bar{\gamma}=|\bar{H}|^{2} T$ holds in deriving the result of Figure 3. Although the probability distribution of average received SNR shown in Figure 3 has similarity to the probability distribution of average channel gain shown in Figure 2, Figure 3 depicts more clearly the influence of $|\bar{H}|^{2}$ on average received SNR. The conclusions that have been drawn from Figure 2 on the differences between BRAN $\mathrm{A}$ and BRAN $\mathrm{C}$ channel models can be observed from Figure 3 more apparently. In Figure 3, the pattern of the probability distribution function defines the effective range of average received SNR. The "effective" range means that the probability distribution of average received SNR falling outside of this range is negligible. From Figure 3 we observe that the effective ranges of average received SNR are different for two channel models. The effective ranges are $[7,22] \mathrm{dB}$ and $[10,21] \mathrm{dB}$

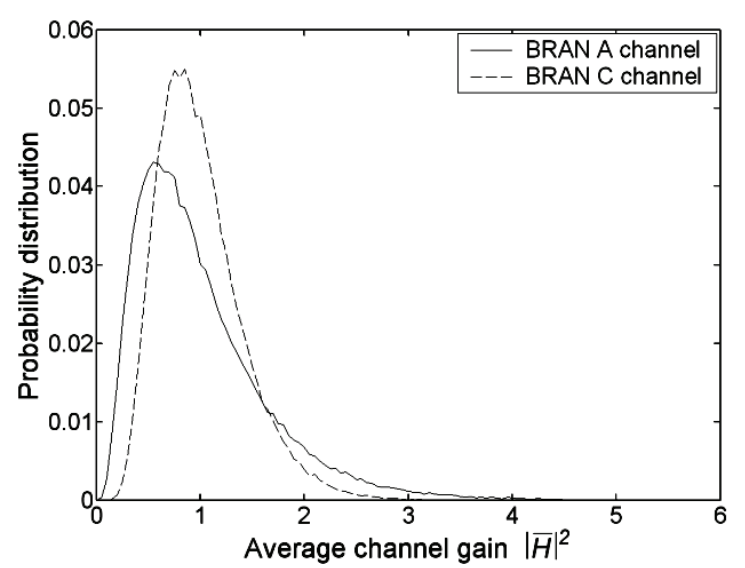

Figure 2. The probability distribution of average channel gain over 100,000 samples of fading realization for BRAN A and $\mathrm{C}$ channel models, respectively.

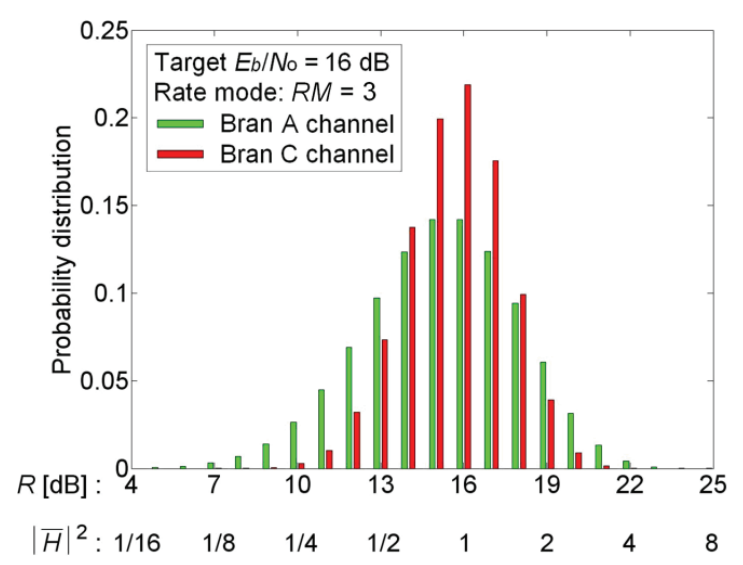

Figure 3. The probability distribution of average received SNR in terms of average channel gain for two channel models given that $R M=3$ and the target $E_{b} / N_{0}$ is $16 \mathrm{~dB}$.

for BRAN A and C channel models, respectively, for $T=$ $16 \mathrm{~dB}$. Clearly, BRAN A channel has longer tails on both sides than BRAN $\mathrm{C}$ channel. Note that if the selected rate mode and the target $E_{b} / N_{0}$ are changed, only the effective ranges of average received SNR are shifted according to (15) but the patterns of the probability distribution for both channel models remain unchanged.

\subsection{PER Calculation for a Received Packet}

The symbol error probability for an $M$-ary QAM [20] with the average SNR per symbol, $s$, can be calculated by

$$
p_{M}(s)=1-\left[1-\left(1-\frac{1}{\sqrt{M}}\right) \operatorname{erfc}\left(\sqrt{\frac{3}{2(M-1)} \cdot s}\right)\right]^{2}
$$


In $802.11 \mathrm{a} / \mathrm{g}$, an OFDM data symbol consists of $N_{S D}$ $=48$ data subcarriers. The average symbol error probability for an $M$-ary QAM over all these data subcarriers can be given by

$$
\bar{p}_{e, M}=\frac{1}{N_{S D}} \sum_{i=1}^{N_{S D}} p_{M}\left(\gamma_{i}\right),
$$

where $\gamma_{i}$ is the received SNR of the $i$-th subcarrier. With a Gray coding, the average bit error probability for an $M$-ary QAM after demodulation is given by

$$
p_{b}=\frac{1}{\log _{2} M} \bar{p}_{e, M} .
$$

In [21], an upper bound was given on the PER under the assumption of binary convolutional coding and harddecision Viterbi decoding with independent errors into the decoder. For a $B$-octet long packet to be transmitted using PHY mode $\phi$, this bound is

$$
p_{e, p k t} \leq 1-\left(1-P_{u}^{\varphi}\right)^{8 B},
$$

where the union bound $P_{u}^{\phi}$ of the first-event error probability is given by, $P_{u}^{\phi}=\sum_{d=d_{\text {free }}}^{\infty} a_{d} P_{d}$ with $d_{\text {free }}$ being the minimum free distance of the convolutional code for the given code rate, $a_{d}$ the total number of error events of weight $d$ [22], and $P_{d}$ the probability of error in the pair-wise comparison of two paths that differ in $d$ bits. When the hard-decision decoding is applied, $P_{d}$ is given by

$$
P_{d}=\left\{\begin{array}{c}
\sum_{k=(d+1) / 2}^{d}\left(\begin{array}{l}
d \\
k
\end{array}\right) p_{b}^{k}\left(1-p_{b}\right)^{d-k}, \\
d=\text { odd } \\
\sum_{k=d / 2+1}^{d}\left(\begin{array}{l}
d \\
k
\end{array}\right) p_{b}^{k}\left(1-p_{b}\right)^{d-k}+\left(\begin{array}{l}
d \\
\frac{d}{2}
\end{array}\right) \frac{p_{b}^{d / 2}\left(1-p_{b}\right)^{d / 2}}{2}, \\
d=\text { even }
\end{array}\right.
$$

where $P_{d}$ is the average bit error probability given by (15).

\subsection{Average PER of an Average Received SNR}

For an $B$-octet long packet to be transmitted using rate mode $\phi$, given that the target $E_{b} / N_{0}$ is $T$, total 100,000 samples of such packet are generated to derive the average PER (an upper bound) of an average received SNR by the following steps.

Step 1: Calculate the initial SNR value per subcarrier using (8).

Step 2: Calculate the average channel gain of each sampling packet using (13).

Step 3: Calculate the average received $\operatorname{SNR}(\bar{\gamma})$ of each sampling packet using (12).

Step 4: Count the number of received packets, denoted by $N_{p k t}(R)$, falling in the given sampling bin belonging to the average received SNR $R$.

Step 5: Calculate the PER of each packet counted in step 4 using (19).

Step 6: The average PER of the average received SNR $R$, denoted by $\overline{P E R}(R)$, is given by

$$
\overline{P E R}(R)=\frac{1}{N_{p k t}(R)} \sum_{i=1}^{N_{p k t}(R)} p_{e, p k t}[i]
$$

where $p_{e, p k t}[i]$ is the PER of the $i$-th packet calculated in step 5.

\subsection{Average PER Performance Comparisons}

Figure 4 presents the performance comparisons of average PER derived in (21) between BRAN A and C channel models for four different rate modes defined in $802.11 \mathrm{a} / \mathrm{g}$, given that $T=16 \mathrm{~dB}$ and $B$ is 196-octet long. The effective range of average received SNR that can be measured by (15) is considered for every PER curve of different rate mode. For example, these ranges are [15, $30] \mathrm{dB}$ and $[18,29] \mathrm{dB}$ for BRAN A and C channels, respectively, given that $R M=8$. From Figure 4 we observe that BRAN $\mathrm{C}$ channel has better performance than BRAN A channel for $R M=1(6 \mathrm{Mb} / \mathrm{s})$ and $R M=3(12 \mathrm{Mb} / \mathrm{s})$ if the values of average received SNR are lower. On the contrary, BRAN A channel outperforms BRAN C channel for $R M=6(36 \mathrm{Mb} / \mathrm{s})$ and $R M=8(54 \mathrm{Mb} / \mathrm{s})$ if the values of average received SNR are higher. These results agree with our observation from Figure 2 and Figure 3

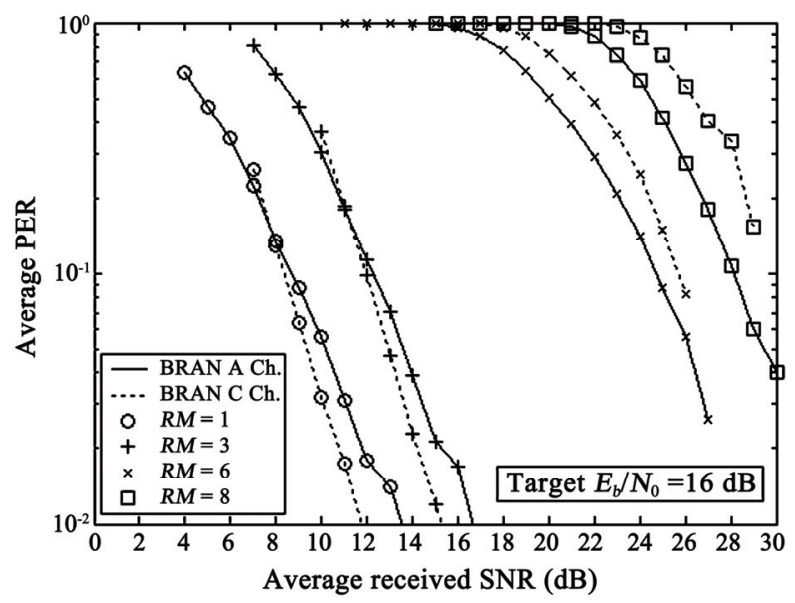

Figure 4. Performance comparisons of average PER between BRAN $A$ and $C$ channels for four different rate modes in $802.11 \mathrm{a} / \mathrm{g}$ given that the target $E_{b} / N_{0}$ is $16 \mathrm{~dB}$. 
that BRAN A channel has higher probability to have smaller values $|\bar{H}|^{2}$ than BRAN C channel. Thus, BRAN A channel degrades PER performance more severely than BRAN C channel as channel conditions are bad. Figure 2 and Figure 3 also show that BRAN A channel has higher probability to have larger values of $|\bar{H}|^{2}$ than BRAN C channel. So that BRAN A channel outperforms BRAN $\mathrm{C}$ channel on PER performance as channel conditions are good.

The results and observations depicted above are rarely found in the literature. Although many researchers have focused on the performance evaluation of different channel models in HiperLAN/2 and 802.11a, such as [12,23], and it is commonly recognized that BRAN channel $\mathrm{C}$ has better performance than channel A. For example, Haider and Raweshidy in [23] concluded that BRAN C channel has better performance than channel A due to the increase of frequency diversity of the channels. Frequency diversity [24] due to delay spread provides the receiver with several (ideally independent) replica of the transmitted signal and is therefore a powerful means to combat fading and interference. Surely BRAN C channel has larger RMS delay spread (150 ns) than that of BRAN A channel (50 ns). The ray (multipath) number of BRAN C channel could be larger than that of BRAN A channel if ideal exponential channel model was considered. But actually both channel models have the same ray number (18-ray) and their PDPs are quite different as shown in Table 2 and Table 3. In this paper, we provide a different approach to compare the average PER performance between two channel models. Our approach provides more insight into the performance differentiation between BRAN A and BRAN C channel models by firstly taking the whole power delay profiles of each channel model into account, secondly by performing performance comparisons on a per target $E_{b} / N_{0}$ basis, and thirdly by considering the effects of the probability distribution of average channel gain on the average PER performances. Our approach for average PER performance comparisons between different channel models can be applied to other channel models as well, on a per target $E_{b} / N_{0}$ basis.

\section{Simulation Model}

Figure 5 shows the simulation model of OFDM-based systems that we used in this paper. The inputs of the simulation model are target $E_{b} / N_{0}$, selected rate mode, and packet length. The output of the simulation model is the estimation of packet error rate. For a given target $E_{b} / N_{0}$ and a certain selected rate mode, the initial SNR value per subcarrier, $\eta$, is obtained by (8). Noise power

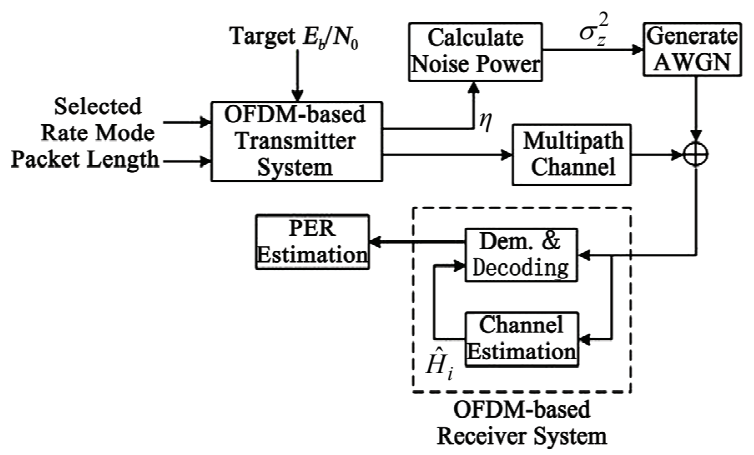

Figure 5. The simulation model of OFDM-based systems.

is calculated by (10) to generate AWGN noise in the simulation. In the IEEE $802.11 \mathrm{a} / \mathrm{g}$ WLAN, there are two long training symbols for each packet that can be used for coherent detection. In this study training-sequence based channel estimation method is used for stationary mobile stations in slowly time-varying environments. The two long training symbols in the PLCP (physical layer convergence procedure) preamble are rate mode independent and are used for the channel estimation of every received PPDU (physical protocol data unit). Other channel estimation methods, such as pilot symbol aided scheme [25-27], and blind estimation technique [28-30], were not considered.

\subsection{Channel Estimation}

After taking an $N$ point FFT at the receiver, the two long training symbols have the same form of the received signal for subcarrier $i$ given by

$$
Y_{n}[i]=F_{i} H_{i}+Z_{n}[i], \quad n=0
$$

where $\left\{F_{i}\right\}$ is the set of training sequence defined in 802.11a standard and is both known by the transmitter and the receiver. In [31], a channel estimation value $\hat{H}_{i}$ of the $i$-th subcarrier in a conventional equalizer can be estimated by

$$
\hat{H}_{i}=\left(Y_{0}[i]+Y_{1}[i]\right) / 2 F_{i},
$$

where $Y_{0}[i]$ and $Y_{1}[i]$ are both complex Gaussian random variables with the same mean $H_{i} F_{i}$. In [32], it has been proved that the optimal estimator of $H_{i}$ is just $\hat{H}_{i}$ defined in (23) by using the likelihood function of $H_{i}$. The likelihood function of $H_{i}$ can be written as

$$
\begin{gathered}
f\left(Y_{0}[i]+Y_{1}[i] ; H_{i}\right) \\
=\frac{1}{\left(2 \pi \sigma_{Z}^{2} / N\right)^{2}} \exp \left[-\frac{1}{2 \sigma_{Z}^{2} / N}\left(\left|Y_{0}[i]-H_{i} F_{i}\right|^{2}\right.\right. \\
\left.\left.+\left|Y_{1}[i]-H_{i} F_{i}\right|^{2}\right)\right] .
\end{gathered}
$$


The optimal estimator of $H_{i}$ is hence

$$
\begin{aligned}
\hat{H}_{i}= & \arg \max _{H_{i}} f\left(Y_{0}[i]+Y_{1}[i] ; H_{i}\right) \\
& =\arg \min _{H_{i}}\left|Y_{0}[i]-H_{i} F_{i}\right|^{2}+\left|Y_{1}[i]-H_{i} F_{i}\right|^{2} \\
& =\left(Y_{0}[i]+Y_{1}[i]\right) / 2 F_{i} .
\end{aligned}
$$

Thus, the estimation of the received SNR $\gamma_{i}$ of the $i$ th subcarrier can be expressed as

$$
\hat{\gamma}_{i}=\left|\hat{H}_{i}\right|^{2} \eta
$$

The estimation of the average channel gain over all subcarriers is given by

$$
|\hat{\bar{H}}|^{2}=\frac{1}{52} \sum_{\text {all } i \in I}\left|\hat{H}_{i}\right|^{2} .
$$

And the estimation of average received SNR of a packet for a certain rate mode is given by

$$
\hat{\bar{\gamma}}=|\hat{\bar{H}}|^{2} T+10 \log _{10}\left(C R \times N_{B P S C}\right) .
$$

\subsection{Analytical Upper Bound and Simulation Results}

In our simulation the size of the physical service data unit (PSDU) is assumed 196-octet long. During transmission, the PSDU is provided with a PLCP preamble and header to create the PPDU. The simulation result of the average PER for a particular average received SNR $R$ is obtained by first counting the number of those received packets (PPDUs) whose estimated average received SNRs falling within the range $(R-0.5, R+0.5]$ but they could not be decoded correctly, and then divided that number by the value of $N_{p k t}(R)$ defined in Subsection 2.4.

Figure 6 shows the results of average PER versus average received SNR for three different values of target $E_{b} /$ $N_{0}$ under BRAN A channel model and $R M=7(48 \mathrm{Mb} / \mathrm{s})$. The results of average PER derived both from the analytical upper bound and the simulation are presented for comparison. From Figure 6, we can see that the results derived from the analytical upper bound and derived from the simulation can be fitted quite well for each case of different target $E_{b} / N_{0}$ values, if the curve derived from the analytical upper bound is shifted leftward by a small amount less than $2 \mathrm{~dB}$. The shifting amounts for $T$ $=22 \mathrm{~dB}, 19 \mathrm{~dB}$ and $16 \mathrm{~dB}$ are $2 \mathrm{~dB}, 1.8 \mathrm{~dB}$ and $1.5 \mathrm{~dB}$, respectively. A similar conclusion concerning the fitting between PER curves derived from the analytical upper bound and derived from the simulation in OFDM-based systems can be found in [2].

In Figure 6, we also see that the PER curve of $T=16 \mathrm{~dB}$ performs the best, and that of the $T=22 \mathrm{~dB}$ performs the worst. This implies better PER performance is obtained with the same average received SNR by lowering the value of target $E_{b} / N_{0}$. This phenomenon has close relationship to the fading effects represented by $|\bar{H}|^{2}$, as illustrated by the following example. For $R M=7$, the average received SNR value of a packet given by (20) becomes $\bar{\gamma}=|\bar{H}|^{2} T+6 \mathrm{~dB}$. For the case of $T=16 \mathrm{~dB}$, the average received SNR will be $25 \mathrm{~dB}$ if we let $|\bar{H}|^{2}$ $=2$. The same average received SNR can be derived if we let $|\bar{H}|^{2}=1$ and $|\bar{H}|^{2}=1 / 2$ for the cases of $T=19$ $\mathrm{dB}$ and $22 \mathrm{~dB}$, respectively. Though the average received SNR for the above three cases are the same, the smallest average PER is obtained by the case of $T=16 \mathrm{~dB}$ under better channel condition $\left(|\bar{H}|^{2}=2\right)$, and the largest average PER is obtained by the case of $T=22 \mathrm{~dB}$ under worse channel condition $\left(|\bar{H}|^{2}=1 / 2\right)$. The impact of this phenomenon on the link adaptation of 802.11 a will be presented in the next section.

\section{Numerical Results and Applications}

For the rate adaptive $802.11 \mathrm{a} / \mathrm{g}$ WLAN systems, this section determines a set of mode switching thresholds for each individual target $E_{b} / N_{0}$ using the results derived above for the average PER, so that the optimal throughput performance can be obtained on a per target $E_{b} / N_{0}$ basis. The lower shifting of mode switching thresholds with the lowering of target $E_{b} / N_{0}$ values is presented by exploiting the phenomenon that we have observed from Figure 6.

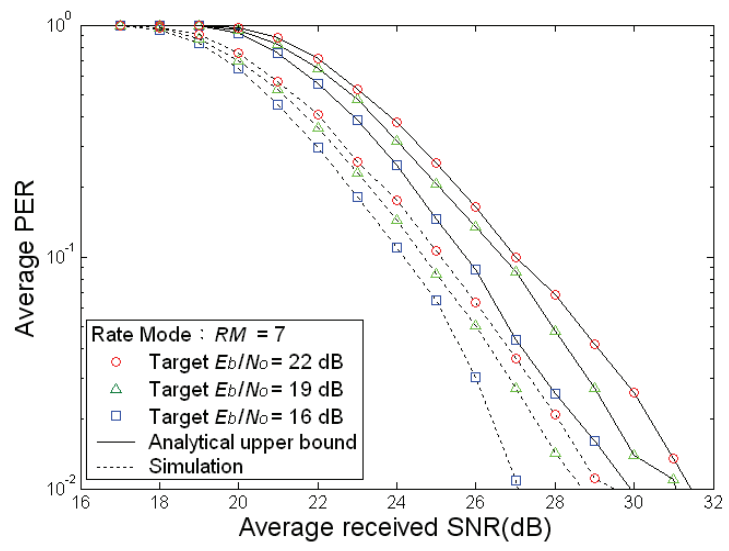

Figure 6. Performance comparisons of average PER between the analytical upper bound and the simulation for three different target $E_{b} / N_{0}$ values under BRAN A channel model and $R M=7$. 


\subsection{Throughput-Based Link Adaptation}

For throughput-based link adaptation, physical link is adapted to the rate mode that gives the highest throughput. For different rate modes used in $802.11 \mathrm{a} / \mathrm{g}$, the relationship between the achieved throughput and the corresponding average PER, denoted by $\overline{P E R}$, can be obtained as follows. During transmission, the PSDU is provided with a PLCP preamble and header to create the PPDU. The transmission time of a PPDU frame (in us) can be calculated as

$$
\begin{aligned}
\mathrm{T}_{\text {PPDU }}= & \mathrm{T}_{\text {PREAMBLE }}(16 \mathrm{us})+\mathrm{T}_{\text {SIGNAL }}(4 \mathrm{us}) \\
& +\mathrm{N}_{\text {DATA_SYM }} \times \mathrm{T}_{\text {SYM }},
\end{aligned}
$$

where $T_{\text {SYM }}$ is the OFDM symbol interval, and $\mathrm{N}_{\text {DATA SYM }}$ is the number of OFDM symbols in the DATA portion of the PPDU frame format and its value is dependent on the data rate used. The value of $\mathrm{N}_{\text {DATA_SYM }}$ is calculated by

$$
\mathrm{N}_{\text {DATA_SYM }}=\left\lceil\frac{16+\text { PSDU_size } \times 8+6}{\mathrm{~N}_{\text {DBPS }}}\right\rceil,
$$

where PSDU_size is the size of the PSDU in bytes, and $\mathrm{N}_{\mathrm{DBPS}}$ is the number of data bits per OFDM symbol which is dependent on the rate mode used. To obtain the net throughput of $802.11 \mathrm{a} / \mathrm{g}$ physical layer, only the payload bits of successful transmitted PPDU frames are considered. Thus, the throughput (in $\mathrm{Mb} / \mathrm{s}$ ) is calculated by

$$
\text { Throughput }=\text { PSDU_size } \times 8 \times(1-\overline{P E R}) / \mathrm{T}_{\mathrm{PPDU}} .
$$

We choose two target $E_{b} / N_{0}$ values $T=12 \mathrm{~dB}$ and $18 \mathrm{~dB}$ to illustrate the throughput-based link adaptation of 802.11 a on a per target $E_{b} / N_{0}$ basis. Average PER derived from the simulation under BRAN A channel model is used for all the cases presented in this section. Both average PER and throughput versus average received SNR for different rate modes under $T=12 \mathrm{~dB}$ and $18 \mathrm{~dB}$ are shown in Figure 7 and Figure 8, respectively. Five contour lines for connecting points where $|\bar{H}|^{2}=$ $1 / 4,1 / 2,1,2$, and 4 on different average PER curves are plotted in Figure 7(a). When compare Figure 7(a) to Figure 8(a), firstly, we notice that contour lines with smaller values of $|\bar{H}|^{2}$ which are absent in Figure 7(a) are now appearing in Figure 8(a); secondly, the contour lines that are of the same values of $|\bar{H}|^{2}$ are shifted right toward higher average received SNR in Figure 8(a). This is because for a particular rate mode to achieve a certain average PER, the channel conditions, represented by $|\bar{H}|^{2}$, experienced by the packets with lower target $E_{b} / N_{0}$ is better than that experienced by the packets with higher target $E_{b} / N_{0}$. For example, to achieve av- erage PER $=10^{-2}$ for $R M=5(24 \mathrm{Mb} / \mathrm{s})$ in Figures 7(a) and 8(a), the corresponding values of $|\bar{H}|^{2}$ are larger than 2 for $T=12 \mathrm{~dB}$ and within $[1 / 2,1]$ for $T=18 \mathrm{~dB}$.

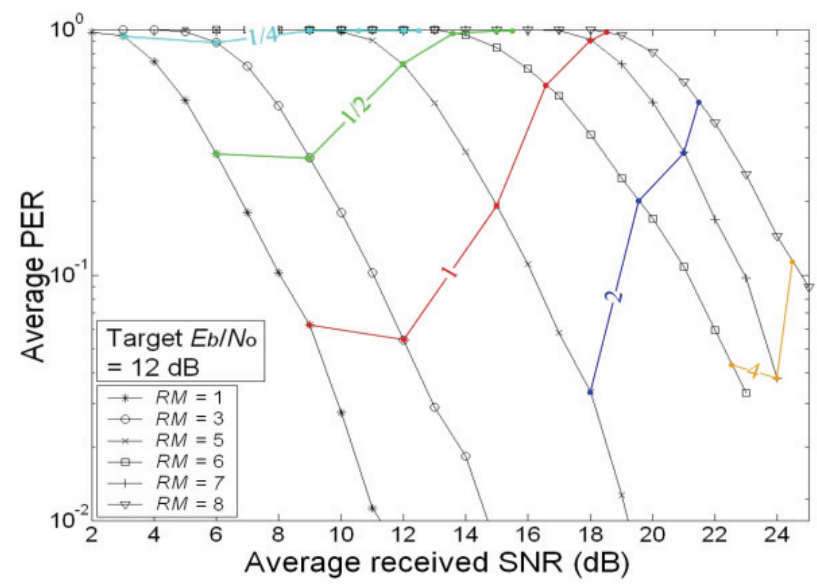

(a)

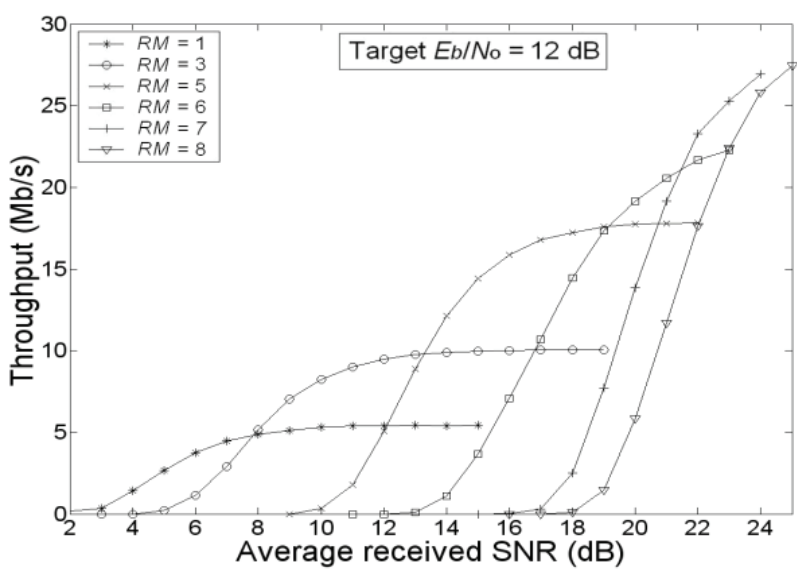

(b)

Figure 7. Throughput-based link adaptation for target $E_{b}$ $/ N_{0}=12 \mathrm{~dB}$.

Figure 7(b) shows the throughput performances of different rate modes under $T=12 \mathrm{~dB}$. Mode switching between neighboring rate modes are clear except that there has no definite rate adaptation between $R M=7$ and $R M=8$. This exception is due to the right-most boundary of the effective ranges of average received SNR for $R M$ $=7$ and $R M=8$ are $24 \mathrm{~dB}$ and $25 \mathrm{~dB}$, respectively. In Figure 7(b), the mode selection ranges for $R M=1$ is $[0$, 7] $\mathrm{dB}$, for $R M=3$ is $[8,13] \mathrm{dB}$, for $R M=5$ is $[14,18] \mathrm{dB}$, for $R M=6$ is $[19,21] \mathrm{dB}$, for $R M=7$ is [22,24], and for $R M=8$ is $[25,-]$. Figure 8(b) shows the result of link adaptation for $T=18 \mathrm{~dB}$. Simulation result shows that there has no need for rate adaptation between $R M=3$ $(12 \mathrm{Mb} / \mathrm{s})$ and $R M=1(6 \mathrm{Mb} / \mathrm{s})$. Therefore the throughput curve for $R M=1$ was not shown in Figure 8(b). This also means that there has no possibility to use $R M=1$ 


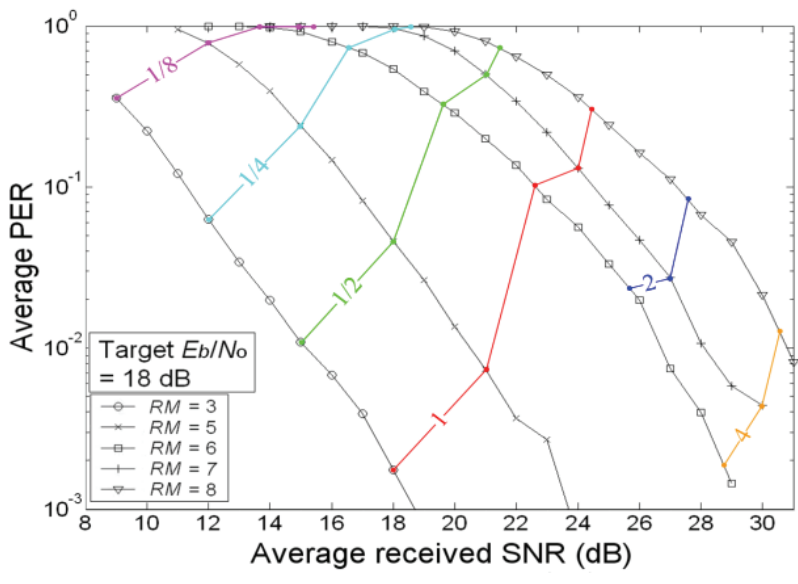

(a)

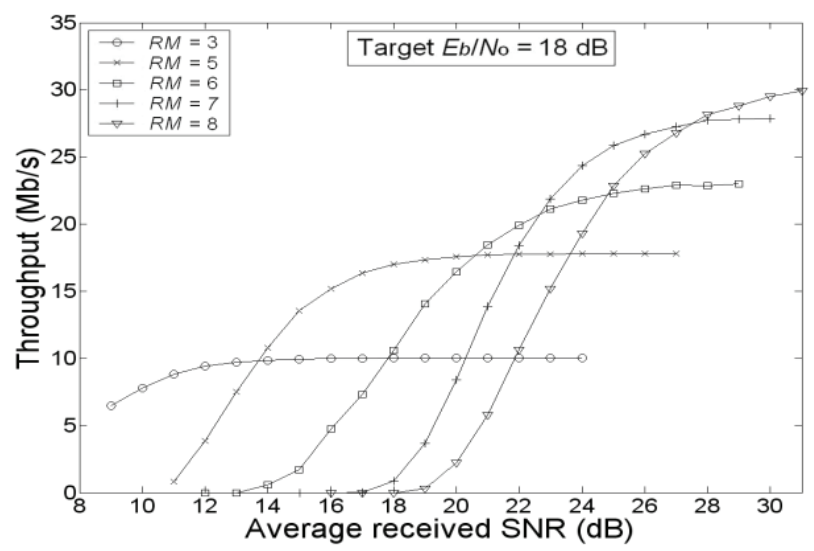

(b)

Figure 8. Throughput-based link adaptation for target $E_{b}$ $/ N_{0}=18 \mathrm{~dB}$.

for $T=18 \mathrm{~dB}$ under BRAN A channel model. In Figure $\mathbf{8}(\mathbf{b})$, the mode selection ranges for $R M=3$ is $[9,13] \mathrm{dB}$, for $R M=5$ is $[14,20] \mathrm{dB}$, for $R M=6$ is $[21,22] \mathrm{dB}$, for $R M=7$ is $[23,27]$, and for $R M=8$ is $[28,31]$. Note that the effective ranges of average received SNR for different rate modes are considered in both Figure 7 and Figure 8. Also in Figure 8, the two PHY rate modes $R M=2$ $(9 \mathrm{Mb} / \mathrm{s})$ and $R M=4(18 \mathrm{Mb} / \mathrm{s})$ were not included. Because in our simulation, the throughput performance of $R M=4$ is worse than that of $R M=3$ and the throughput performance of $R M=2$ is worse than that of $R M=1$.

\subsection{Lower Shifting of Mode Switching Thresholds}

As we have observed from Figure 6 that better PER performances with the same average received SNR are obtained by lowering the values of target $E_{b} / N_{0}$. By exploiting this phenomenon this subsection presents the lower shifting of mode switching thresholds with the lowering of target $E_{b} / N_{0}$ values. An example is given as below for illustrative purpose. Consider the results of average PER for $R M=7$ in both Figures 7(a) and 8(a). For $R M=7$, we have already known that $\bar{\gamma}=|\bar{H}|^{2} T+$ $6 \mathrm{~dB}$. For the case of $T=12 \mathrm{~dB}$, the average received SNR will be $21 \mathrm{~dB}$ if we let $|\bar{H}|^{2}=2$. The same average received SNR is obtained if we let $|\bar{H}|^{2}=1 / 2$ for the case of $T=18 \mathrm{~dB}$. From Figure 7(a), we see that the average PER of average received $\mathrm{SNR}=21 \mathrm{~dB}$ is about 0.3 for $R M=7$ under $T=12 \mathrm{~dB}$. However, from Figure 8(a) we observe that this value is about 0.5 under $T=18$ dB. Accordingly, the achieved net throughputs of average received $\mathrm{SNR}=21 \mathrm{~dB}$ for $R M=7$ are about $19 \mathrm{Mb} / \mathrm{s}$ and $14 \mathrm{Mb} / \mathrm{s}$ under $T=12 \mathrm{~dB}$ and $T=18 \mathrm{~dB}$, respectively, as that shown in Figures 7(b) and 8(b). Therefore we have shown that by lowering the value of $T$, better throughput performance with the same average received SNR can be achieved; consequently, rate adaptation to the next lower rate mode can be shifted downwardly. Compare the mode switching thresholds between $R M=6$ and $R M=7$ in Figures 7(b) and $\mathbf{8}(\mathbf{b})$, we see that this threshold is shifted downward from $22 \mathrm{~dB}$ under $T=18$ dB to $21 \mathrm{~dB}$ under $T=12 \mathrm{~dB}$.

Figure 9 provides a full view of the lower shifting of mode switching thresholds for the link adaptation of 802.11a over BRAN A channel model under different target $E_{b} / N_{0}$ values. In Figure 9, the $\mathrm{X}$-axis denotes the average received SNR and the $\mathrm{Y}$-axis denotes the desired target $E_{b} / N_{0}$. Six rate modes are considered in the rate adaptation of $802.11 \mathrm{a}$. The set of mode switching thresholds for target $E_{b} / N_{0}=T \mathrm{~dB}$ can be obtained from Figure 9 by drawing a horizontal line on $T$ (Y-axis) and finding the intersections between this horizontal line and the vertical lines (mode switching boundaries). For every given target $E_{b} / N_{0}$, the effective range of average received SNR is confined by the cross points plotted on the both sides. The cross point on the right-hand side is determined by the effective range of the highest rate mode that the given target $E_{b} / N_{0}$ may achieve; and the cross point on the left-hand side is determined by the effective range of the lowest rate mode that the given target $E_{b} / N_{0}$ may reach. Ideally, for every given value of $T$ there should have five mode switching thresholds corresponding to all the pairs of neighboring rate modes. However, this may not be the case due to the rate modes that can be adapted to under a specific value of $T$ may not cover all six rate modes. For example, the set of mode switching thresholds under $T=18 \mathrm{~dB}$ is $[-, 13,20$, $22,27] \mathrm{dB}$ since there has no rate adaptation between $R M$ $=1$ and $R M=3$.

An interesting phenomenon that can be observed from 
Figure 9 is that the lower shifting of mode switching thresholds occurred more frequently between neighboring rate modes $R M=7$ and $R M=8$ and also between $R M=5$ and $R M=6$. Coincidentally, each pair of neighboring rate modes has the same and high modulation level. The first pair, $R M=7$ and $R M=8$, uses the same modulation scheme 64 QAM, and the second pair, $R M=5$ and $R M=$ 6 , uses the same modulation scheme 16 QAM. The lower shifting of mode switching thresholds occurred less frequently between neighboring rate modes with different modulation levels, for example $R M=3$ with QPSK and $R M=5$ with 16 QAM. Moreover, there has no lower shifting of mode switching thresholds between $R M=1$ with BPSK and $R M=3$ with QPSK. More investigations are needed to further understand this phenomenon.

\subsection{System Throughput Optimization}

In this subsection, we show that no other sets of mode switching thresholds can achieve higher system throughput than the optimal set which is determined on a per target $E_{b} / N_{0}$ basis. The following example is given for illustrative purpose. From Figure 9, we have the optimal set of mode switching thresholds under $T=18 \mathrm{~dB}$ is $[-, 13,20$, $22,27] \mathrm{dB}$. Here we purposely choose two other sets of mode switching thresholds $[-, 16,23,25,29] \mathrm{dB}$ and $[-, 12$, $18,20,24] \mathrm{dB}$. The thresholds in the first set are tending towards right, so we called them upward-shifted thresholds. On the contrary, the thresholds in the second set are tending towards left, so we called them downward-shifted thresholds. The comparisons of achieved system throughputs between the optimal set and these two sets of mode switching thresholds under $T=18 \mathrm{~dB}$ are presented in Figures 10(a) and 10(b), respectively. In Figure 10(a), the throughput achieved by the optimal set of mo-

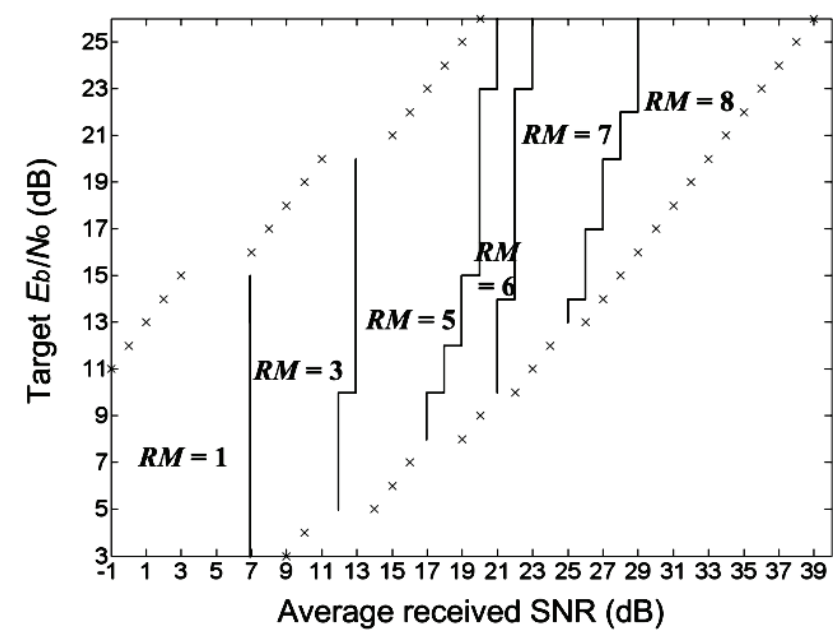

Figure 9. A full view of the lower shifting of mode switching thresholds for the link adaptation of 802.11a over BRAN A channel model under different target $E_{b} / N_{0}$ values. de switching thresholds is expressed by the solid curves, and that achieved by the upward-shifted thresholds is expressed by the dotted curves. Obviously, the solid curves are always higher than or equal to the dotted curves for every average received SNR. In Figure 10(b) we observe that rate adaptations using downward-shifted thresholds always occurred at smaller average received SNR values than that using the optimal thresholds. So that solid curves are also higher than or equal to dotted curves for every average received SNR in Figure 10(b).

\section{Conclusions}

For the rate adaptive $802.11 \mathrm{a} / \mathrm{g}$ WLAN, an analytical approach is proposed to obtain the average PER of individual average received SNR value for different rate modes on a per target $E_{b} / N_{0}$ basis. Instead of just looking

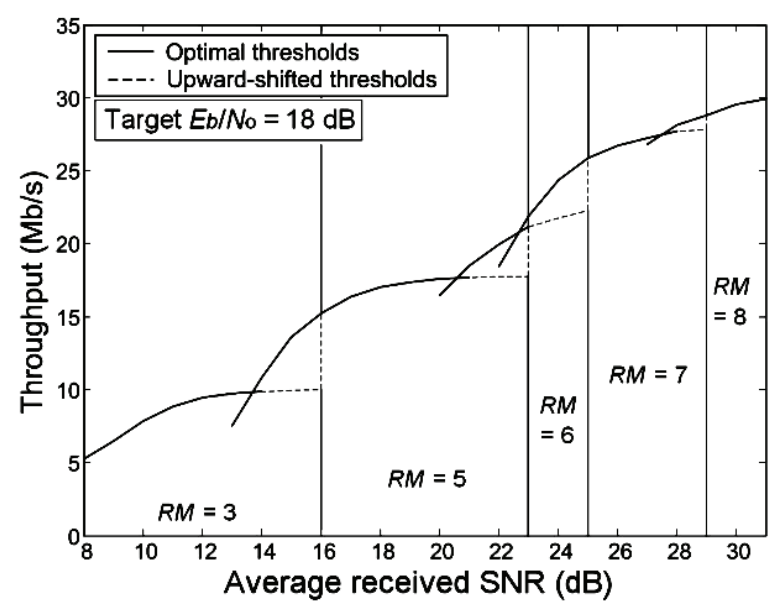

(a)

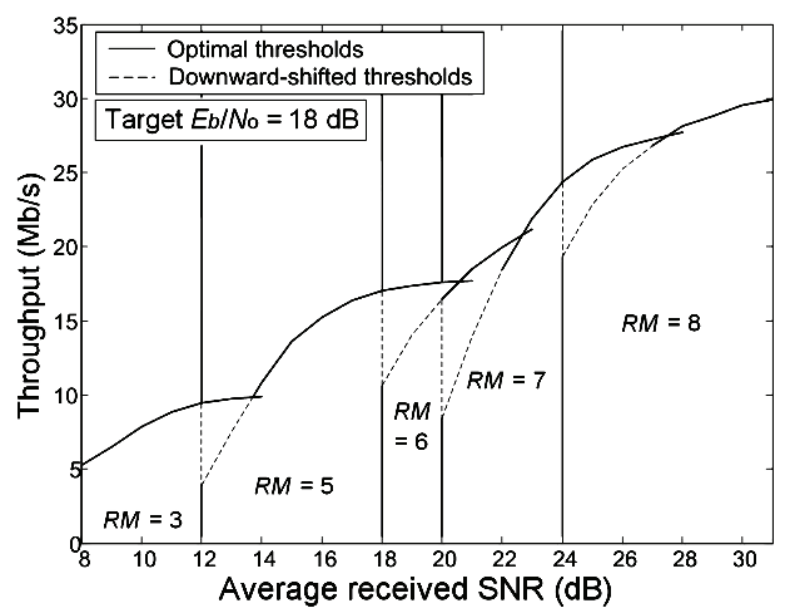

(b)

Figure 10. The comparisons of achieved system throughputs between the optimal set and the other two sets of mode switching thresholds: (a) Upward-shifted thresholds; (b) Downward-shifted thresholds, under $T=18 \mathrm{~dB}$. 
at the average received SNR value of a packet, this paper considers the whole distribution of the average received SNR, which leads to a better average PER and throughput performances. In this paper we also showed that the probability distribution of average channel gain can affect the average PER over the effective range of average received SNR for different rate modes on a per target $E_{b} / N_{0}$ basis. Furthermore, we have shown that better PER performances with the same average received SNR can be obtained by lowering the values of target $E_{b} / N_{0}$. By exploiting this phenomenon, the mode switching thresholds for optimal throughput performance of each target $E_{b} / N_{0}$ can be determined. Numerical results show that mode switching thresholds can be shifted downwards with the lowering of target $E_{b} / N_{0}$ values. A full view of the lower shifting of mode switching thresholds for the link adaptation of 802.11a over BRAN A channel model under different target $E_{b} / N_{0}$ values is presented. From this an interesting phenomenon is observed, that is, the lower shifting of mode switching thresholds can occur more frequently between neighboring rate modes with the same and high modulation level - e.g. 64 QAM or 16 QAM. The lower shifting of mode switching thresholds occurred less frequently between neighboring rate modes with different modulation levels - e.g. QPSK and 16 QAM. Further investigations are needed to have a better understanding of this phenomenon.

\section{Acknowledgements}

This work was supported by the National Science Council, Taiwan, under the contracts NSC 96-2221-E-224002-MY2.

\section{References}

[1] IEEE Standard 802.11a, Part 11: Wireless LAN Medium Access Control (MAC) and Physical Layer (PHY) Specifications: High-speed Physical Layer in the 5GHz Band, Supplement to the IEEE 802.11 Standard, September 1999.

[2] O. Awoniyi and F. A. Tobagi, "Packet Error Rate in OFDM-Based Wireless LANs Operating in Frequency Selective Channels," Proceedings of 25th IEEE International Conference on Computer Communications, Barcelona, 23-29 April 2006, pp. 1-13.

[3] J. Armstrong, "Analysis of New and Existing Methods of Reducing Intercarrier Interference Due to Carrier Frequency Offset in OFDM," IEEE Transactions on Communications, Vol. 47, No. 3, March 1999, pp. 365-369.

[4] W. Zhang and J. Lindner, "SINR Analysis for OFDMA Systems with Carrier Frequency Offset," Proceedings of 18th IEEE International Symposium on Personal, Indoor and Mobile Radio Communications, Athens, 3-7 Septem- ber 2007, pp. 1-5.

[5] Q. Yang, F. Fu, Y. Wu and K. S. Kwak, "Sub-Optimum Superimposed Training Design for Estimating of Mobile OFDM Channels," Proceedings of 4th International Conference on Communications and Networking in China, Xi'an, 26-28 August 2009, pp. 1-5.

[6] M. Lampe, H. Rohling and W. Zirwas, "Misunderstandings about Link Adaptation for Frequency Selective Fading Channels," Proceedings of 13th IEEE International Symposium on Personal, Indoor and Mobile Radio Communications, Lisbon, Vol. 2, 15-18 September 2002, pp. 710-714.

[7] M. Lampe, T. Giebel, H. Rohling and W. Zirwas, "PERPrediction for PHY Mode Selection in OFDM Communication Systems," Proceedings of IEEE Global Telecommunications Conference, San Francisco, Vol. 1, 1-5 December 2003, pp. 25-29.

[8] C. Snow, L. Lampe and R. Schober, "Error Rate Analysis for Coded Multicarrier Systems over Qusai-Static Fading Channels," IEEE Transactions on Communications, Vol. 55, No. 9, September 2007, pp. 1736-1746.

[9] H. Gong, W. Ye, S. Feng and H. Song, "A Subcarrier Allocation Algorithm for Efficiently Reducing Power in Multiuser OFDM Systems," Wireless Personal Communications, Vol. 40, No. 2, 2007, pp. 233-243.

[10] J. Medbo and P. Schramm, "Channel Models for HIPERLAN/2,” ETSI/BRAN Document Number 3ERI085B, 1998.

[11] D. Qiao, S. Choi and K. G. Shin, "Goodput Analysis and Link Adaptation for IEEE 802.11a Wireless LANs," IEEE Transactions on Mobile Computing, Vol. 1, No. 4, October 2002, pp. 278-292.

[12] S. Armour, A. Doufexi, A. Nix and D. Bull, "A Study of the Impact of Frequency Selectivity on Link Adaptive Wireless LAN Systems," Proceedings of 56th IEEE Vehicular Technology Conference, Vancouver, Vol. 2, 24-28 September 2002, pp. 738-742.

[13] L. B. Le, E. Hossain and M. Zorzi, "Queueing Analysis for GBN and SR ARQ Protocols under Dynamic Radio Link Adaptation with Non-Zero Feedback Delay," IEEE Transactions on Wireless Communications, Vol. 6, No. 9 , September 2007, pp. 3418-3428.

[14] B. O'Hara and A. Petrick, "IEEE 802.11 Handbook-A Designer's Companion,” IEEE Press, Hoboken, 1999.

[15] M. R. Raghavendra, S. Bhashyam and K. Giridhar, "Fast Multipath Delay Estimation in OFDM Systems Using Frequency Swept Pilots," Proceedings of 9th International OFDM Workshop, Dresden, 15-16 September 2004, pp. 237-240.

[16] N.-L. Hung, L.-N. Tho and C. C. Ko, "Joint Channel Estimation and Synchronization with Inter-Carrier Interference Reduction for OFDM," Proceedings of IEEE International Conference on Communications, Glasgow, 24 28 June 2007, pp. 2841-2846.

[17] J. Tao, J. Wu and C. Xiao, "Channel Estimation for OFDM Systems in the Presence of Carrier Frequency Offset and Phase Noise," Proceedings of IEEE International Con- 
ference on Communications, Beijing, 19-23 May 2008, pp. 5072-5076.

[18] H. Kang, W. Hwang and K. Kim, "OFDM Systems with Subchannel Power Control under the Two-Ray Multipath Channel," Proceedings of IEEE International Conference on Communications, Helsinki, Vol. 6, 11-14 June 2001, pp. 1856-1860.

[19] M. S. Bahaei, "Joint Optimization of Transmission Rate and Outer-Loop SNR Target Adaptation over Fading Channels," IEEE Transactions on Communications, Vol. 55, No. 3, March 2007, pp. 398-403.

[20] S. Haykin, "Communication Systems," 4th Edition, John Wiley \& Sons, Hoboken, 2001.

[21] M. B. Pursley and D. J. Taipale, "Error Probabilities for Spread-Spectrum Packet Radio with Convolutional Codes and Viterbi Decoding," IEEE Transactions on Communications, Vol. 35, No. 1, January 1987, pp. 1-12.

[22] D. Haccoun and G. Begin, "High-Rate Punctured Convolutional Codes for Viterbi and Sequential Decoding," IEEE Transactions on Communications, Vol. 37, No. 11, November 1989, pp. 1113-1125.

[23] K. Haider and H. S. Al-Raweshidy, "Evaluation of User Capacity and Channel Model Effect in HiperLAN/2 System," Proceedings of 4th International Workshop on Mobile and Wireless Communications Network, Stockholm, 9-11 September 2002, pp. 554-558.

[24] H. Bolcskei and A. J. Paulraj, "Space-Frequency Coded Broadband OFDM Systems," Proceedings of IEEE Wireless Communications and Networking Conference, Chicago, Vol. 1, 23-28 September 2000, pp. 1-6.

[25] M. Morelli and U. Mengali, "A Comparison of PilotAided Channel Estimation Methods for OFDM Systems," IEEE Transactions on Signal Processing, Vol. 49, No. 12, December 2001, pp. 3065-3073.
[26] R. Gruenheid, H. Rohling, J. Ran, E. Bolinth and R. Kern, "Robust Channel Estimation in Wireless LANs for Mobile Environments," Proceedings of 56th IEEE Vehicular Technology Conference, Vancouver, Vol. 3, 24-28 September 2002, pp. 1545-1549.

[27] Z. Yuanjin, "A Novel Channel Estimation and Tracking Method for Wireless OFDM Systems Based on Pilots and Kalman Filtering," IEEE Transactions on Consumer Electronics, Vol. 49, No. 2, May 2003, pp. 275-283.

[28] A. P. Petropulu, R. Zhang and R. Lin, "Blind OFDM Channel Estimation through Simple Linear Precoding," IEEE Transactions on Wireless Communications, Vol. 3, No. 2, March 2004, pp. 647-655.

[29] F. Gao and A. Nallanathan, "Blind Channel Estimation for OFDM Systems via Generalized Precoding," IEEE Transactions on Vehicular Technology, Vol. 56, No. 3, May 2007, pp. 1155-1164.

[30] F. Gao, Y. Zeng, A. Nallanathan and T. S. Ng, "Robust Subspace Blind Channel Estimation for Cyclic Prefixed MIMO OFDM Systems: Algorithm, Identifiability and Performance Analysis," IEEE Journal on Selected Areas in Communications, Vol. 26, No. 2, February 2008, pp. 378-388.

[31] H.-W. Kim, C.-H. Lim and D.-S. Han, "Viterbi Decoder Aided Equalization and Sampling Clock Tracking for OFDM WLAN," Proceedings of 60th IEEE Vehicular Technology Conference, Los Angeles, Vol. 5, 26-29 September 2004, pp. 3738-3742.

[32] W.-C. Liu, L.-C. Wang and Y.-W. Lin, "Physical Layer Effects on the MAC Goodput Performance for the Rate Adaptive IEEE 802.11a/g WLAN," Proceedings of IEEE Wireless Communications and Networking Conference, Atlanta, Vol. 3, 21-25 March 2004, pp. 1873-1878. 